\title{
Rotavirus Double and Triple Layered Viral Particles: Correlative Characterization Using Electron Microscopy, Disc centrifuge and Capillary Electrophoresis
}

\author{
A. Miseur ${ }^{1}$, P. Blain ${ }^{1}$, A. Coppens ${ }^{1}$, C. Chapelle ${ }^{1}$, C. Hens ${ }^{1}$, G. Delpierre ${ }^{1}$ and M. Deschuyteneer ${ }^{1}$. \\ ${ }^{1 .}$ GSK Vaccines, Analytical R\&D, rue de l'Institut, 89, Rixensart, Belgium.
}

Rotavirus (Reoviridae) viral particles consist of capsids with 3 concentric protein layers surrounding the virus genetic material. The outer layer is composed of the VP4 and VP7 proteins, which independently elicit neutralizing antibodies and induce protective immunity.

Rotarix $^{\mathrm{TM}}$, a live attenuated rotavirus vaccine, has been characterized by EM. Both negative staining (Fig. 1) and cryo-EM (Fig. 2) were applied. The observations showed that the vaccine contained both triple-layered particles (TLPs) containing all 3 protein layers and double-layered particles (DLPs) which lack the outer capsid proteins VP4 and VP7. Their apparent diameter difference of about $10 \mathrm{~nm}$ was well correlated in both techniques.

As DLPs are non-infectious, it may be of importance to address the question of their abundance $v s$ TLPs in bulk preparations. Although such information can be obtained by EM, the method is relatively impractical for this purpose, particularly for routine analysis, as high number of particles should be observed and sized to get accurate relative abundances.

To determine the size and quantify the relative abundance of TLPs and DLPs on large populations of viral particles in bulk preparations, two size-based, orthogonal analytical techniques were applied to detect these particles: capillary electrophoresis (CE) and disc centrifuge (DC). To confirm the capacity of both methods to distinguish clearly the two types of capsids, TLPs and DLPs were partially purified using $\mathrm{CsCL}_{2}$ gradient. The profiles (Fig. 3) show the well-resolved identification.

In addition, further confirmation was obtained by incubation of the preparations with EDTA, to remove the calcium required for VP7 protein stabilisation and thus to convert TLPs into DLPs ${ }^{1}$. The shift from TLPs to DLPs was clearly visible with CE (not shown), DC and also EM (Fig. 4). Dynamic Light Scattering (DLS) was also evaluated and worked fine with the partially purified samples of either TLPs or DLPs. The shift from TLPs to DLPs by this method was clearly observed as well. However, DLS did not allow to distinguish clearly the two populations of capsids in real samples with mixed TLPs and DLPs (data not shown).

These orthogonal structural and sizing analysis methods can be combined to assess and measure accurately the DLPs/TLPs ratio. They provide a tool to understand the possible link of this ratio with the immunological potency of vaccine preparations.

References:

[1] Mathis PK et al, Journal of Virological Methods 169, p. 13.

[2] Rotarix is a trademark of the GSK group of companies. 


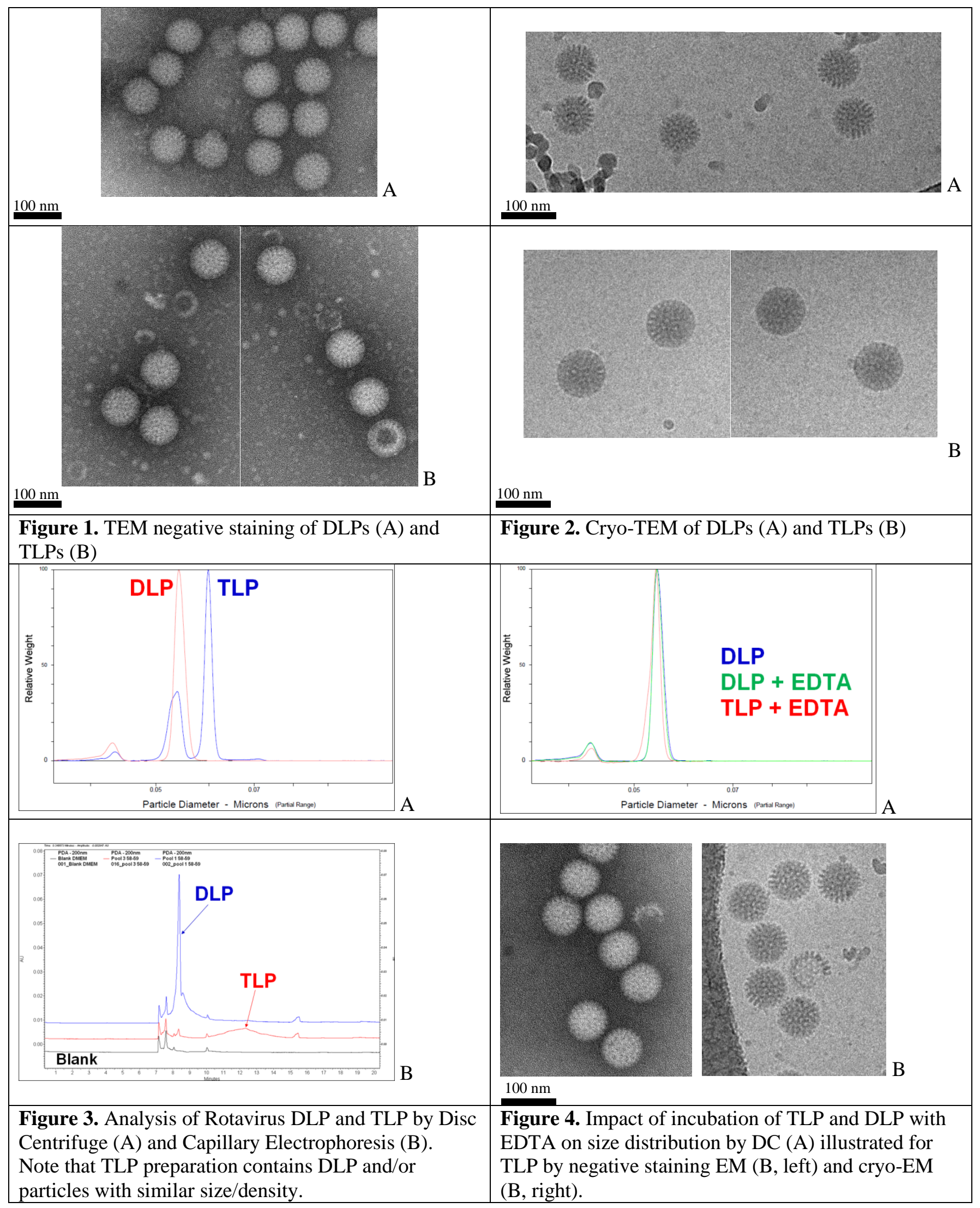

